\title{
Technical Advances in the Field of ECMO
}

\author{
Peter Betit MBA RRT RRT-NPS FAARC
}

\author{
Introduction \\ Artificial Gas Exchange \\ ECMO Systems \\ Extracorporeal $\mathrm{CO}_{2}$ Removal \\ Respiratory Dialysis \\ Wearable ECMO \\ Simulation-Based Training \\ Summary
}

\begin{abstract}
Although the fundamentals of extracorporeal membrane oxygenation (ECMO) have not changed in 3 decades, the technical elements continue to improve and have evolved from an assemblage of individual components to more integrated systems with added features, enhanced safety, and improved maneuverability. The introduction of polymethylpentene (PMP) fiber technology has expanded the development of artificial membranes that have low resistance, are more biocompatible, and can be used for extended durations. Extracorporeal carbon dioxide removal techniques continue to be enhanced as stand alone technology and modified renal dialysis systems are introduced. Research continues in the development of compact and wearable artificial lungs that are intended to support patients for prolonged periods (eg, patients awaiting lung transplantation). The use of high-fidelity simulation training has become a standard and important method for reinforcing technical skills, refining troubleshooting sequences, and enhancing team interactions. Modifications to mannequins and ECMO systems coupled with clinical and physiologic scenarios will help achieve greater realism and enhance learning. ECMO technology continues to improve, with adaptability and versatility being essential attributes. Key words: extracorporeal membrane oxygenation; polymethylpentene; centrifugal pump; respiratory dialysis; decarboxylation; high-fidelity simulation. [Respir Care 2018;63(9):1162-1173. (C) 2018 Daedalus Enterprises]
\end{abstract}

\section{Introduction}

Extracorporeal membrane oxygenation (ECMO) has been a vital tool in the management of severe cardiorespiratory dysfunction across all patient ages for more than 3 decades. As of 2015, > 78,000 cases have been reported

The author has disclosed no conflicts of interest.

Correspondence: Peter Betit MBA RRT RRT-NPS FAARC, Boston Children's Hospital, Respiratory Care / ECMO Program,300 Longwood Ave, Boston, MA 02115. E-mail: peter.betit@childrens.harvard.edu.

DOI: $10.4187 /$ respcare.06320 to the Extracorporeal Life Support Organization, and the number of centers reporting to this international database, now exceeding 300 globally, has steadily increased. ${ }^{1}$ The use of ECMO in adults with cardiac and respiratory conditions has been the category with the largest growth, with $>2,000$ cases reported in 2015 and a 57\% survival to hospital discharge. ${ }^{1}$ This growth is supported by ongoing research in techniques including low-flow extracorporeal $\mathrm{CO}_{2}$ removal and variations such as respiratory dialysis and wearable long-term thoracic artificial lungs. ${ }^{2}$ While the fundamentals of ECMO have not significantly changed, the components that comprise an ECMO circuit have become more integrated and streamlined, are relatively easy to assemble and prepare, and have advanced features and enhanced 


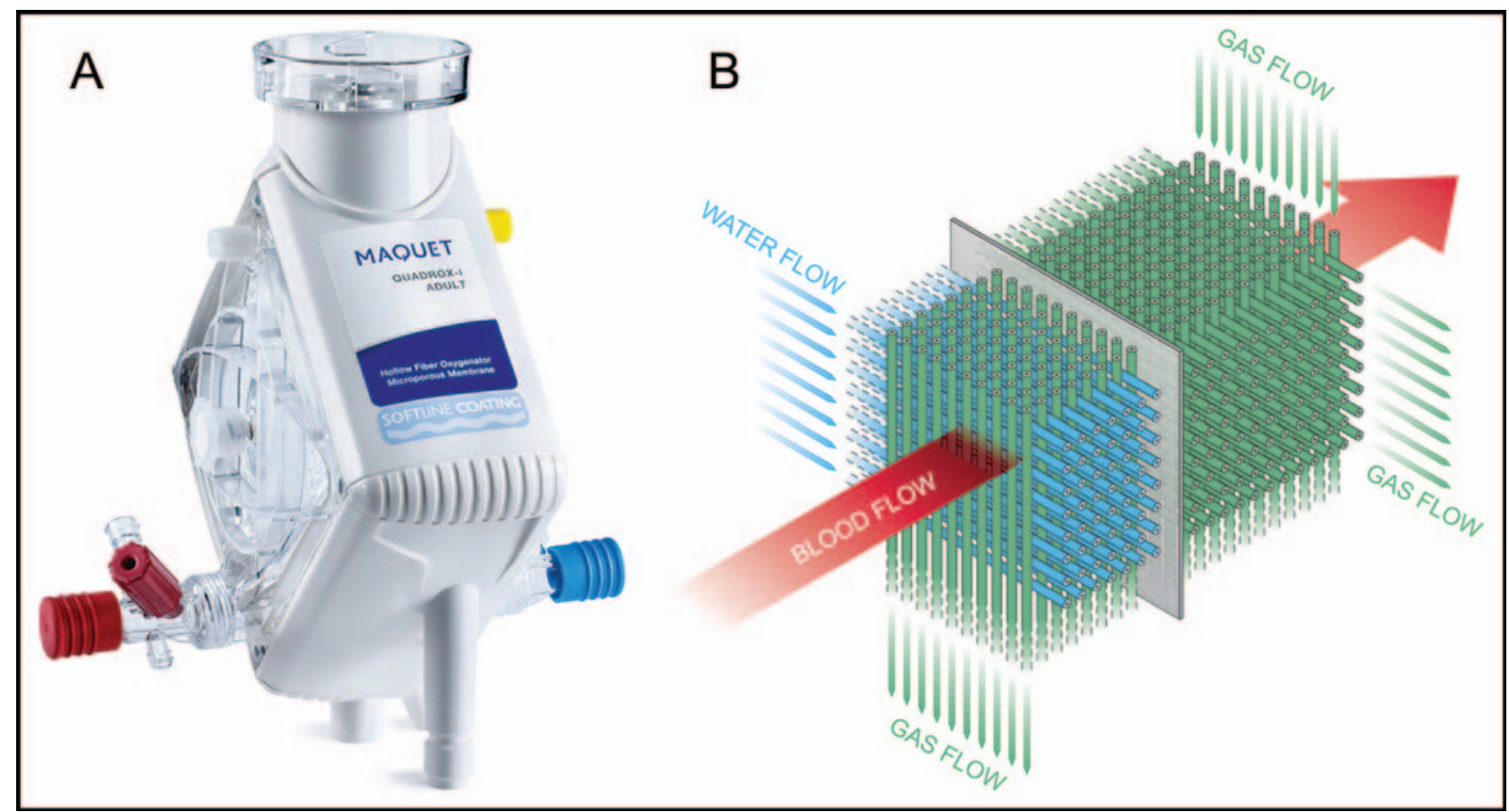

Fig. 1. The Quadrox-iD (A) and cross-section of diffusion membrane showing blood, water, and gas flow through a mat of hollow fibers (B). Courtesy of Maquet.

safety mechanisms. ${ }^{3}$ The availability of polymethylpentene (PMP) fiber technology has led to a new generation of efficient and low-resistance artificial gas-exchange membranes. ${ }^{4}$ The development of more integrated and realistic simulation equipment has enhanced interdisciplinary team training. ${ }^{5}$ The aim of this paper is to review the technical developments in ECMO technology, including devices and techniques that may transition from bench to bedside.

\section{Artificial Gas Exchange}

The extracorporeal exchange of oxygen and $\mathrm{CO}_{2}$ occurs by simple diffusion within an artificial lung or membrane oxygenator, which contains bundles of artificial capillaries or hollow fibers that serve as channels for blood, gas, and water. ${ }^{6}$ Fresh gas is directed into the lumen of the fibers, while blood passes externally and thermo-regulated water flows through adjacent channels. ${ }^{7}$ These hollow-fiber membranes have been used for several decades in both shortterm cardiopulmonary bypass and long-term extracorporeal support. Hollow fibers have typically been constructed of polypropylene, but this material is microporous and, when used for extended periods, it has a tendency to become increasingly hydrophilic due to lipoprotein absorption. This can lead to plasma leakage (also known as plasma wetting), which is the seeping of plasma into the gas compartment of the membrane and can occur within $8 \mathrm{~h}$ of use and can affect membrane performance. ${ }^{4,8}$ Despite this limitation, some
ECMO programs have incorporated microporous hollow-fiber membranes into their systems due to their low resistance and ease of preparation, developing troubleshooting steps to replace them when plasma leakage occurred.

Advances in hollow-fiber membrane engineering and design include the use of fibers constructed with PMP, a polymer that has an asymmetric pore structure and outer skin which permits gas permeability but inhibits plasma leakage. ${ }^{4}$ The brand of fibers principally used to construct today's hollow-fiber membranes is Oxyplus (Membrana, Wuppertal, Germany), now a division of the 3M Industrial Business Group.

The Quadrox-iD (Maquet, Rastatt, Germany) was the first so-called plasma-tight membrane oxygenator made available in the United States, and it was quickly adopted by ECMO centers (Fig. 1). The architecture of the fiber bundles is a unique interwoven mat with channels for blood, gas, and water running perpendicular to each other. This arrangement of fibers differs from most oxygenators, in which fibers are configured in a winding or spiral pattern with channels running more parallel. Purported benefits of the Quadrox-iD, available in adult and pediatric sizes, include efficient gas and temperature transfer rates, low pressure gradient, ability to rapidly prime and de-air, and integral heat exchanger.

Recently the Medos Hilite LT (Xenios, Heilbronn, Germany), one of the first PMP oxygenators used in Europe, became available in the United States (Fig. 2). This mem- 


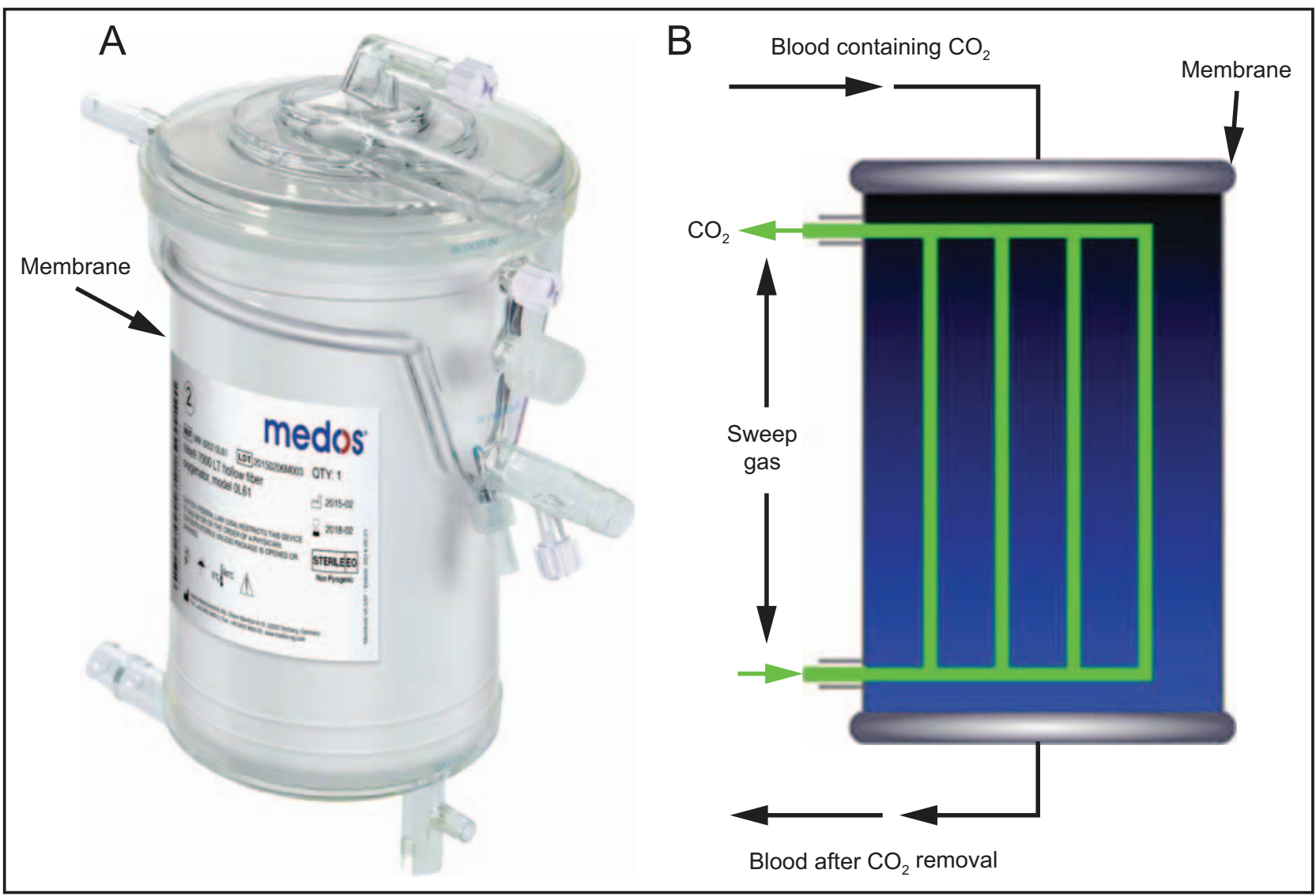

Fig. 2. Image of the Medos Hilite LT (A) and diagram of flow through blood and sweep gas channels (B). Courtesy of Xenios.

brane comes in 3 sizes, has a cylindrical shape, and has attributes similar to the Maquet device. Additional devices with equivalent characteristics and gas transfer profiles are also available outside of the United States. Few studies have compared the clinical performance of these oxygenators, with device selection often based on availability and cost. In one study, the Medos Hilite LT was found to have greater oxygen transfer rates and $\mathrm{CO}_{2}$ removal relative to surface area compared to the Quadrox-iD Pediatric. ${ }^{9}$ This study was insufficiently powered to determine the superiority of a particular device. Other limitations included variable anticoagulation management or other subject factors that may have affected membrane performance.

Contemporary long-term oxygenators marketed as being plasma-tight have been a key advancement in ECMO technology. There is one case report of a plasma-tight membrane experiencing plasma leakage described by Gill et al, ${ }^{10}$ who observed a collection of fluid at the gas egress of a Medos Hilite LT oxygenator. As this fluid accumulated, an increase in fresh gas flow was required due to retained $\mathrm{CO}_{2}$ and presumed failing gas permeability. Once the membrane was replaced, the gas flow was reduced to previous levels and no further plasma wetting was noted. The oxygenator was investigated by the manufacturer and end users, who found no integrity issues with the fibers. The clinicians speculated that it was the patient's condition that altered the protein layer of the membrane and thus caused the leakage, and they suggested that the oxygenator is microporous under certain conditions.

Eash and colleagues ${ }^{8}$ conducted an in vitro experiment to determine the extent of plasma resistance and gas permeability in a variety of experimental skinned fibers as well as the clinically used Membrana (ie, Oxyplus). This experiment used techniques to force wetting-out and suggested that fibers which are more plasma-tight had reduced gas permeability, in particular to $\mathrm{CO}_{2}$. The Membrana fibers had the highest degree of wetting-out under these extreme conditions, but the authors concluded that all of the fibers tested had suitable plasma resistance for clinical use. It is not clear whether plasma leakage is a prevalent clinical concern during ECMO, but it should be recognized that while contemporary PMP membranes may be resistant to plasma leakage, they are not necessarily leak-proof.

\section{ECMO Systems}

Contemporary ECMO systems incorporate non-occlusive centrifugal pump technology due to the advantages of 


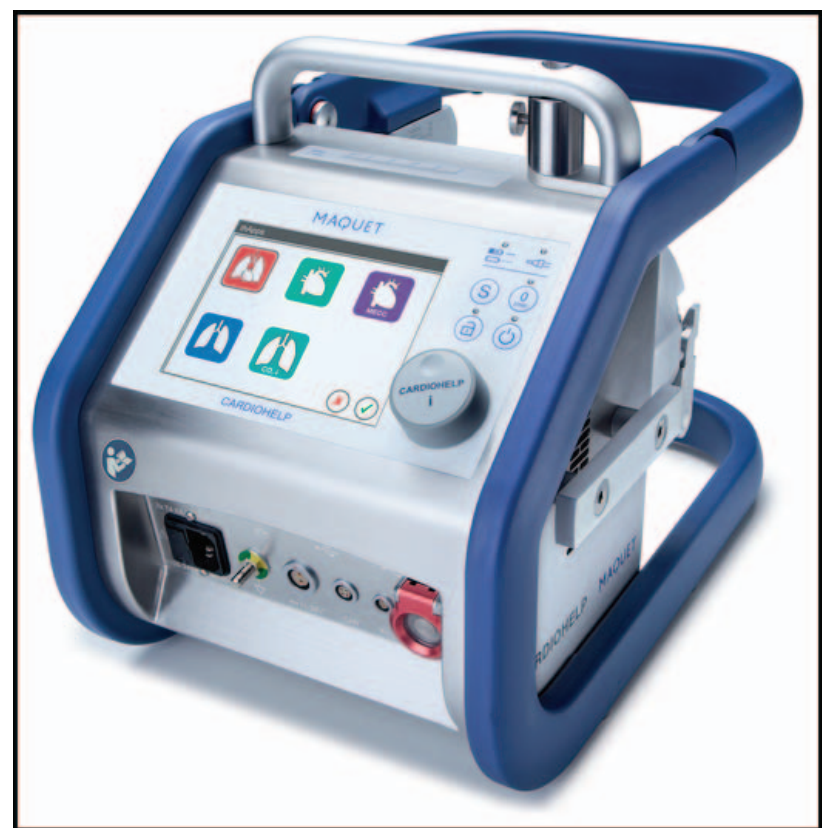

Fig. 3. Cardiohelp, a compact and integrated extracorporeal membrane oxygenation system. Courtesy of Maquet.

smaller priming volumes, streamlined circuitry, and potentially less risk of air emboli. ${ }^{11}$ This style of rotary pump has been used in $60 \%$ of pediatric applications despite the debate that it may be more hemolytic than historicallyused occlusive roller pumps. ${ }^{11}$ Current centrifugal pump technology includes pump heads that rotate on a single pivot point, which results in less friction and a more freefloating mechanism with improved flow characteristics and purported minimal blood cell damage. Stand alone centrifugal pumps such as the Rotaflow (Maquet) have been routinely used in ECMO programs in the United States for well over a decade. ${ }^{12}$

The Cardiohelp (Maquet) is a state-of-the-art miniaturized ECMO system with a uniquely coupled centrifugal pump and PMP membrane (Fig. 3). ${ }^{13}$ This combined system contains integrated sensors and transducers that provide continuous monitoring of system pressures, temperature, flow, venous oxygen saturation, hemoglobin, and hematocrit. The component is secured to a relatively lightweight driver that controls pump speed, contains various configurations and safety features, and provides a continuous readout of all monitored parameters. The Cardiohelp was initially introduced to the United States market in 2012 and is increasingly being used by ECMO programs.

In a cohort of 22 adults with respiratory failure, the Cardiohelp was found to effectively correct blood gasexchange derangements and improve hemodynamics and overall subject stability. ${ }^{14}$ The authors suggested that the biocompatibility of the circuit would result in fewer thromboembolic complications and lessen the need for blood-

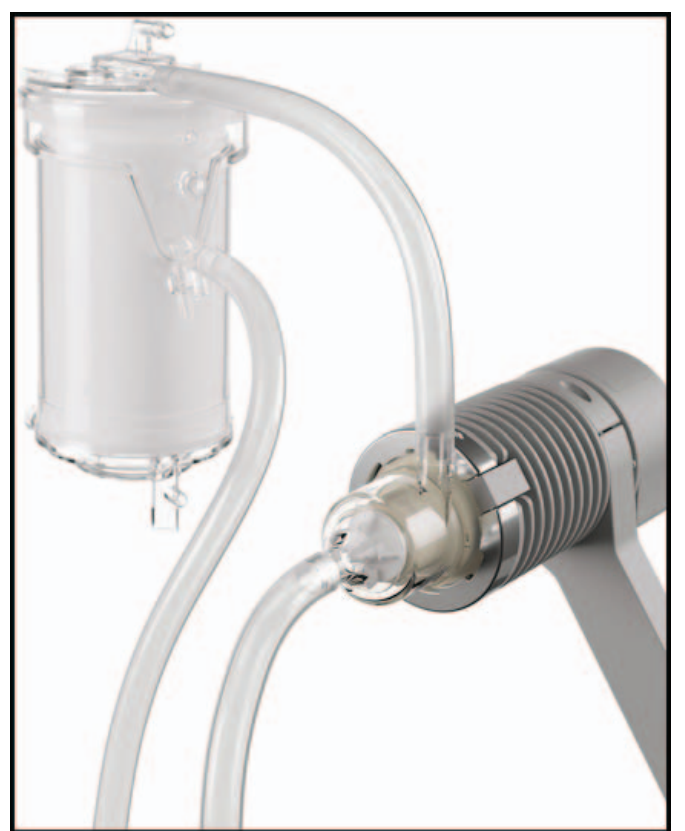

Fig. 4. The Deltrastream DP3 pump and driver with Medos Hilite LT hollow-fiber membrane. Courtesy of Xenios.

product repletion. In 9 of the 22 subjects, the device was replaced due to clot burden in the circuit. Because of the integration of all elements into a single component, an entire circuit exchange is required, which may lead to increased program expenses; in addition, it was noted that the cost of a Cardiohelp device exceeded conventional, less integrated ECMO systems.

The Cardiohelp has been a welcomed advancement in the realm of inter-facility ECMO transport. Its compact size is its principle advantage, but its weight of approximately $9 \mathrm{~kg}$ and ease of maneuverability are beneficial features as well. Improved monitoring and safety features during ground and air transport were advantages noted by Philipp et al. ${ }^{15}$ In a retrospective comparison of the Cardiohelp versus a less integrated ECMO system, Alwardt and colleagues ${ }^{13}$ described a number of advantages of the Cardiohelp, including less adjunct equipment required, simplified circuitry, a user-friendly interface, and ease of preparation and deployment.

Advances in rotational pump design include diagonal pumps in which the impellers that move blood through the pump head do so in a diagonally streamed manner. One such device is the Deltastream DP3 (Xenios) (Fig. 4). ${ }^{16}$ Features of this technology include a low prime volume of $16 \mathrm{~mL}$, a compact design, the capability to generate up to 10,000 revolutions/min, and flows to support the full range of patients with an upper limit of $8 \mathrm{~L} / \mathrm{min}$ (Fig. 4). In a case series of 16 pediatric ECMO subjects, the DP3 was found to be safe and effective and with a low degree of hemolysis. ${ }^{16}$ The lightweight and compact design of this 
device has been found to be suitable for inter-facility ECMO transport by helicopter. ${ }^{17}$

The versatility of the DP3 system was highlighted in a cohort of 233 pediatric subjects across 7 centers. ${ }^{18}$ In this series, the DP3 was used for a variety of mechanical circulatory support applications, including venous-venous ECMO, venous-arterial ECMO, and as a ventricular assist device, with indications equally distributed between cardiac and respiratory support. There was a $26 \%$ rate of equipment exchanges-membrane or complete systemacross the 7 centers and with variable rationales ranging from routine to emergent. This case series demonstrated that the DP3 has multifunctional usefulness, was easy to prepare and handle, and was capable of supporting the full range of mechanical circulatory support indications. In another single-center case series, Speth et al ${ }^{19}$ supported 27 pediatric subjects and emphasized the device's safety features, such as the ability to prevent retrograde flow, and the system's adaptability.

An additional attribute of the DP3 is the availability of a pulsatile mode. In an in vitro study, the DP3 was shown to produce adequate pulsatile flow across small-bore cannulas typically used in neonates; 20 in addition, despite the added resistance of a membrane and circuitry, the DP3 was found to generate adequate pulsatility over a wide range of flows. ${ }^{21}$ Similarly, pulsatile flow was found to be sufficient in a pediatric model, with greater hemodynamic energy created over the alternate non-pulsatile mode. ${ }^{22}$ Pulsatile flow may also be synchronized with the patient's electrocardiogram, similar to the mechanisms of an aortic balloon pump. ${ }^{23}$ An evaluation of this option suggested that the DP3 can produce sufficient pulsatile flow at various simulated heart rates, ratios, and arrhythmias. ${ }^{23}$ It is unclear whether a pulsatile feature would significantly improve outcomes in patients receiving long-term ECMO support, but when initiating extracorporeal cardiopulmonary resuscitation, it seems that a pulsatile feature could potentially improve coronary perfusion. ${ }^{21}$ There is also the potential for this mode to lessen the dampening of pulsatility produced by the native cardiac output and improve perfusion to end organs. ${ }^{21}$

\section{Extracorporeal $\mathrm{CO}_{2}$ Removal}

Extracorporeal $\mathrm{CO}_{2}$ removal, also referred to as arteriovenous $\mathrm{CO}_{2}$ removal, is the technique by which an artificial lung is placed between an arterial-to-venous connection typically established through femoral vessels. ${ }^{24}$ In this pumpless configuration, the patient's native cardiac output provides the driving pressure for blood, which flows through the membrane oxygenator by means of the arterial-to-venous shunt. The aim of this approach is to augment $\mathrm{CO}_{2}$ removal so that lung-protective ventilation can be assured. While pumpless extracorporeal $\mathrm{CO}_{2}$ removal has been shown to improve outcomes in a few case series, it has also been associated with limb ischemia because femoral vessels were principally used.

Pump-driven extracorporeal $\mathrm{CO}_{2}$ removal has emerged as another means of providing effective $\mathrm{CO}_{2}$ removal, and it is typically achieved with a dual-lumen cannula inserted in the right internal jugular or femoral vein. ${ }^{24}$ One system developed by Novalung (a subsidiary of Xenios) utilizes the DP3 and a PMP diffusion membrane minus the integral heat exchanger; this method is known as interventional lung assist or interventional assist membrane ventilator and is marketed as the Novalung iLA Active (Inspiration Healthcare, Crawley, United Kingdom). This approach was used in 12 adult subjects (6 with hypercapnic lung failure and the remaining with hypoxic respiratory failure) and was found to effectively remove $\mathrm{CO}_{2}$ and correct respiratory acidosis, thus permitting the use of lungprotective ventilation. ${ }^{25}$ There was a $60 \%$ survival rate in this preliminary study, with a pretreatment ventilator duration in excess of 2 weeks associated with non-survival. Generally, this study confirmed that pump-driven extracorporeal $\mathrm{CO}_{2}$ removal can efficiently reduce $\mathrm{CO}_{2}$ levels and lessen ventilator-induced lung injury, but that identification of the ideal candidate for this minimally invasive support will require further investigation.

The variables that control the rate of $\mathrm{CO}_{2}$ clearance during extracorporeal $\mathrm{CO}_{2}$ removal are the fresh gas or sweep gas supplied to the membrane and blood flows. In a small cohort of subjects, $\mathrm{CO}_{2}$ removal was greater when blood flow was held constant and sweep gas was increased as demonstrated by a drop in $\mathrm{P}_{\mathrm{aCO}}{ }_{2}{ }^{26}$ The sweep gas range used was $1-14 \mathrm{~L} / \mathrm{min}$, which corresponded to a decrease in the median $\mathrm{P}_{\mathrm{aCO}_{2}}$ from $66 \mathrm{~mm} \mathrm{Hg}$ to $49 \mathrm{~mm} \mathrm{Hg}$ without any appreciable effect on $\mathrm{P}_{\mathrm{aO}}$. When the sweep gas was held constant at a median rate of $4 \mathrm{~L} / \mathrm{min}$ and blood flow was increased from $0.5 \mathrm{~L} / \mathrm{min}$ to $2.0 \mathrm{~L} / \mathrm{min}$, there was a similar effect on $\mathrm{CO}_{2}$ removal but a greater effect on median $\mathrm{P}_{\mathrm{aO}}$, which increased from $67 \mathrm{~mm} \mathrm{Hg}$ to $117 \mathrm{~mm} \mathrm{Hg}$. The authors suggested that the use of higher blood flows may require the use of high negative pressure generated on the drainage side of the circuit, which could lead to hemolysis.

One possible benefit of pump-driven extracorporeal $\mathrm{CO}_{2}$ removal is the prevention of intubation and mechanical ventilation and the avoidance of associated complications. In a feasibility and safety study, the Novalung was used in 25 subjects with COPD, of whom 14 did not require intubation. ${ }^{27}$ Eleven subjects progressed to intubation, 7 primarily due to hypoxemia and 4 for progressive respiratory failure. The extracorporeal $\mathrm{CO}_{2}$ removal cohort was paired with a control group of 25 subjects who received standard treatment. The goal of avoiding intubation was accomplished in half of the study group, and there were no 


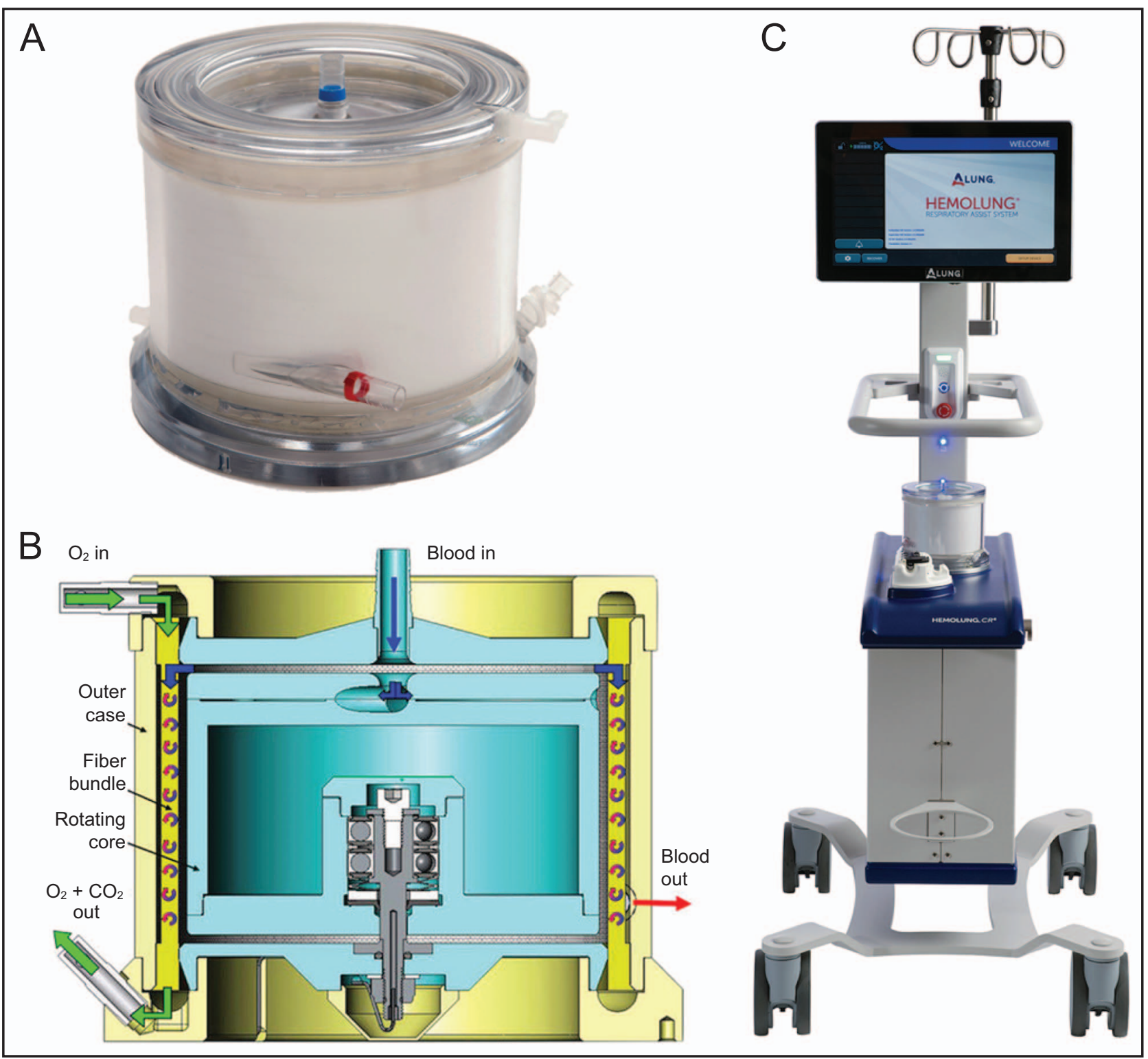

Fig. 5. Hemolung core with rotating pump and fiber bundle (A), cross-sectional diagram (B), and the console and monitoring platform (C). Courtesy of Alung Technologies.

significant differences between the 2 groups with respect to length of stay or mortality.

The Hemolung (ALung Technologies, Pittsburgh, Pennsylvania) is a recent innovation in the field of low-flow extracorporeal $\mathrm{CO}_{2}$ removal. ${ }^{28}$ This device couples gas exchange and blood flow in a single cartridge and is intended as an adjunct respiratory support for patients with acute hypercapnic respiratory failure (Fig. 5). Blood is distributed to a ring of bundled hollow fibers by means of a rotary pump situated in the core, and sweep gas is pulled through the system by a vacuum mechanism. The cartridge is situated in a driver, which is controlled by a user interface and is arranged as a stand alone device. The system is connected to the patient by a 15.5-Fr dual-lumen catheter that is placed in the internal jugular or femoral vein (Fig. 6). Low blood flows of $300-500 \mathrm{~mL} / \mathrm{min}$ are used to further augment $\mathrm{CO}_{2}$ removal. ${ }^{29}$

In a small study, 20 subjects with respiratory failure associated with COPD were supported with the Hemolung. ${ }^{30}$ Prior to initiation of support, the subjects were either receiving noninvasive ventilation, had a high probability of requiring intubation and invasive ventilation or already required invasive ventilation, and had persistent hypercapnic acidosis as determined by a $\mathrm{pH}<7.25$ and $\mathrm{P}_{\mathrm{aCO}_{2}}>55 \mathrm{~mm} \mathrm{Hg}$. The dual-lumen catheter was placed in the right internal jugular vein in 13 subjects, and fem- 


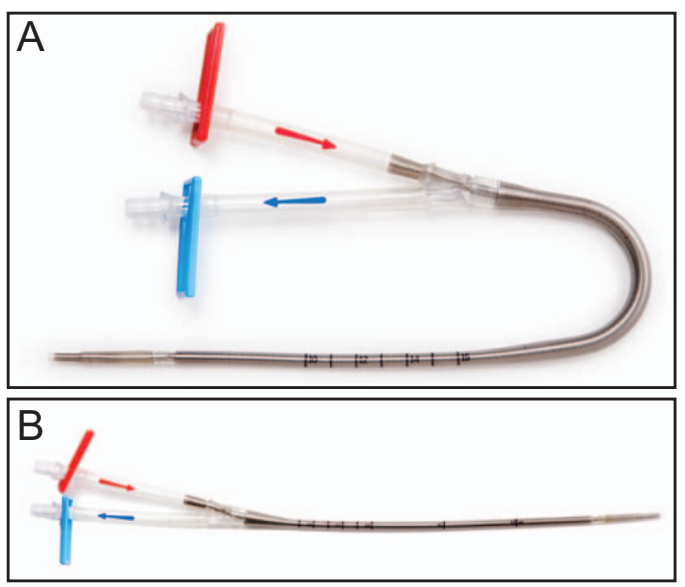

Fig. 6. Hemolung 15.5-Fr dual-lumen jugular (A) and femoral catheters (B). Courtesy of Alung Technologies.

oral access was used in the remainder. Within $1 \mathrm{~h}$ of starting support, $\mathrm{P}_{\mathrm{aCO}}$ levels decreased from a mean of $79 \mathrm{~mm} \mathrm{Hg}$ to $66 \mathrm{~mm} \mathrm{Hg}$, and a corresponding improvement in $\mathrm{pH}$ from 7.28 to 7.34 was observed (Fig. 7). The Hemolung consistently removed $80 \mathrm{~mL} / \mathrm{min}$ of $\mathrm{CO}_{2}$, and complications were mainly related to catheter placement. Intubation was not required in the subjects who were receiving noninvasive ventilation, and overall there was clinical improvement in this cohort. The results of this study confirmed the feasibility of utilizing low-flow extracorporeal $\mathrm{CO}_{2}$ removal with the Hemolung and demonstrated effective $\mathrm{CO}_{2}$ removal. The authors acknowledged that determining the optimal patient population for this modality will require ongoing research, including a randomized, controlled trial.

In a small case series, the Hemolung was found to effectively augment $\mathrm{CO}_{2}$ removal in subjects with acute or acute-on-chronic hypercapnic respiratory failure. ${ }^{31}$ The range of blood flow was $450-550 \mathrm{~mL} / \mathrm{min}$ and sweep gas was increased to $10 \mathrm{~L} / \mathrm{min}$, achieving $\mathrm{CO}_{2}$ removal of 90-100 $\mathrm{mL} / \mathrm{min}$. This permitted the reduction of ventilator support as demonstrated by a decrease in peak inspiratory pressure delivered by a ventilator and an improvement in minute ventilation.

Low-flow extracorporeal $\mathrm{CO}_{2}$ removal with the Hemolung was used in a series of adult subjects with moderate ARDS with the goal of achieving ultra-protective lung ventilation, which included the use of tidal volumes in the range of $4-6 \mathrm{~mL} / \mathrm{kg}^{32} \mathrm{In}$ this 15 -subject series, the Hemolung was connected to the dual-lumen catheter and initiated at a flow of $350-500 \mathrm{~mL} / \mathrm{min}$, but the sweep gas was left at $0 \mathrm{~L} / \mathrm{min}$. Tidal volumes were then reduced to $4 \mathrm{~mL} / \mathrm{kg}$ after $2 \mathrm{~h}$, and when hypercapnia occurred (ie, at $\mathrm{pH}<7.25$ and $\mathrm{P}_{\mathrm{aCO}_{2}}>60 \mathrm{~mm} \mathrm{Hg}$ ), the sweep gas was titrated upward to $10 \mathrm{~L} / \mathrm{min}$. This strategy permitted the
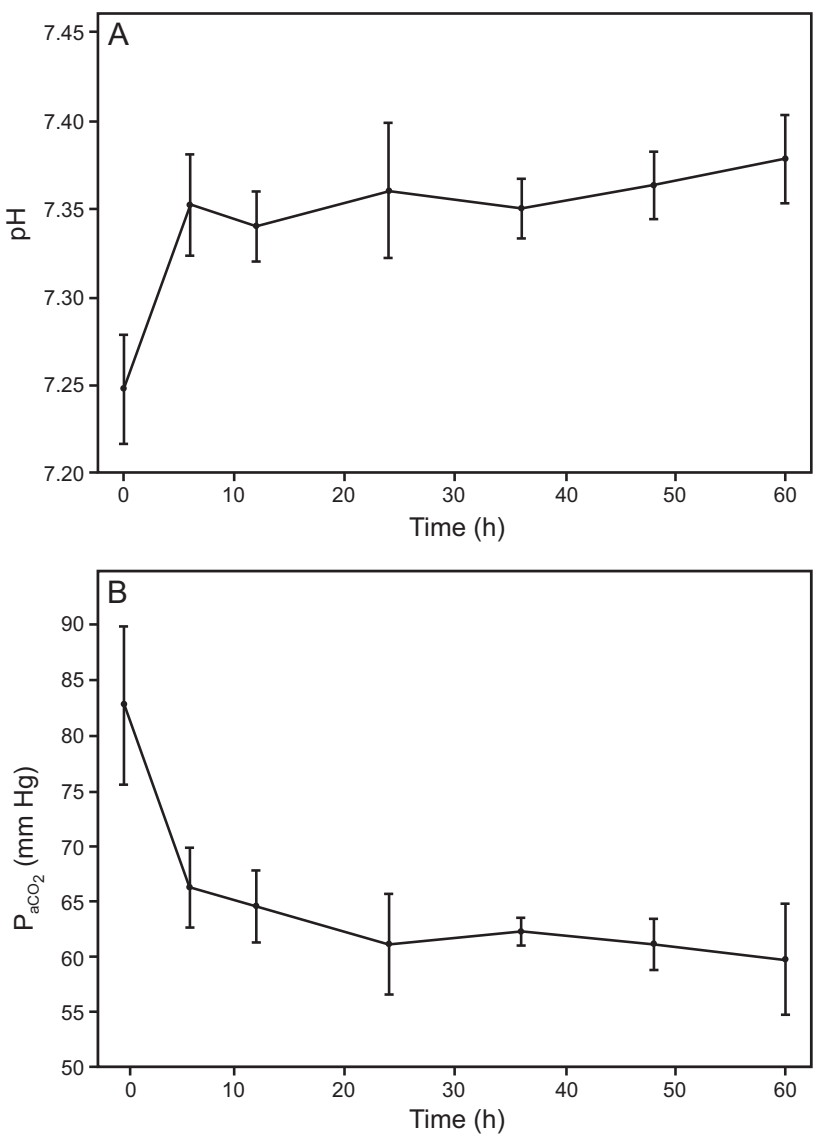

Fig. 7. Changes in mean $\mathrm{pH}$ and $\mathrm{P}_{\mathrm{acO}}$ in subjects with COPD exacerbation receiving noninvasive ventilation. From Reference 30 , with permission.

reduction of tidal volumes to $4 \mathrm{~mL} / \mathrm{kg}$ by correcting the associated respiratory acidosis.

The use of a pump to provide low-flow extracorporeal $\mathrm{CO}_{2}$ removal with devices like the Novalung and the Hemolung has emerged as a viable, safe, and effective means of regulating $\mathrm{CO}_{2}$ removal in patients with hypercapnic respiratory failure such that lung-protective ventilation strategies can be employed. This has been demonstrated in case series, but additional research is needed to determine which population of patients will benefit most from this approach, when in the patients' course it should be used, and the risk-to-benefit ratio given that vascular access and anticoagulation are required.

\section{Respiratory Dialysis}

Extracorporeal $\mathrm{CO}_{2}$ removal devices like the Hemolung operate at blood flows similar to those used with hemodialysis and continuous renal replacement therapy (CRRT) systems, and they have been referred to as respiratory dialysis. ${ }^{33}$ This concept of respiratory dialysis has prompted experiments that utilize CRRT platforms to serve as the 


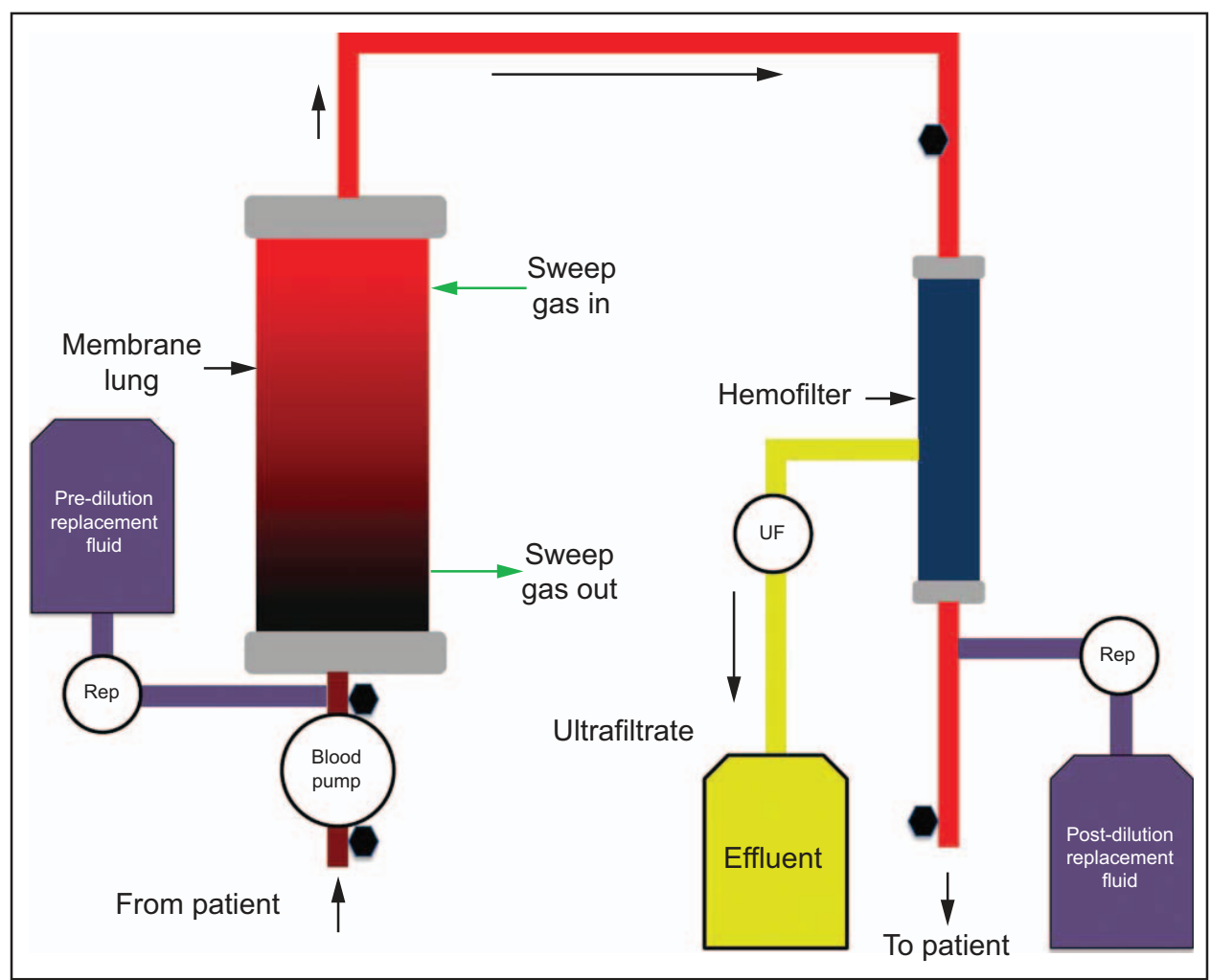

Fig. 8. Schematic representation of a hollow-fiber membrane positioned in series with a hemofilter with a renal replacement therapy platform. Circles show system pumps and hexagons represent the required pressure sensors. Rep = replacement fluid pump; UF = ultrafiltration pump. From Reference 33, with permission.

pump for the gas-exchange membrane (Fig. 8). Interposing an infant-size Medos Hilite LT membrane (Xenios) onto a standard CRRT device minus the hemofiltration cartridge, Godet et al ${ }^{34}$ demonstrated sufficient decarboxylation with a $46 \%$ reduction in $\mathrm{P}_{\mathrm{CO}_{2}}$ and concomitant increase in $\mathrm{pH}$ by 0.24 . Results of this animal study suggest that CRRT devices may be more readily available and could be modified to provide pump-driven extracorporeal $\mathrm{CO}_{2}$ removal.

Similarly, the Abylcap system, consisting of a Lilliput 2 hollow-fiber membrane (LivaNova, Italy) integrated into the Lynda dialysis system (Bellco Medtronic, Mirandola, Italy), was used to provide extracorporeal $\mathrm{CO}_{2}$ removal in 4 subjects with ARDS by means of a 13.5-Fr femoral catheter. ${ }^{35}$ Over a 5-d course, $\mathrm{P}_{\mathrm{CO}}$ had decreased by about $38 \%$, permitting a reduction in the median tidal volume from $6.4 \mathrm{~mL} / \mathrm{kg}$ to $4.8 \mathrm{~mL} / \mathrm{kg}$ and in plateau pressure from $32 \mathrm{~cm} \mathrm{H}_{2} \mathrm{O}$ to $19 \mathrm{~cm} \mathrm{H}_{2} \mathrm{O}$. Increasing blood flow from $200 \mathrm{~mL} / \mathrm{min}$ to $400 \mathrm{~mL} / \mathrm{min}$ enhanced $\mathrm{CO}_{2}$ clearance with sweep gas flows of $6 \mathrm{~L} / \mathrm{min}$. This device was further evaluated in a prospective study of 10 subjects with ARDS who were unresponsive to standard treatment. ${ }^{36}$ In this study ventilator settings were left at baseline while support was initiated so that short-term effects could be assessed; during this period, $\mathrm{CO}_{2}$ was reduced by about $20 \%$. The device was used for a median duration of $6 \mathrm{~d}$ while lung-protective ventilation was provided.

The provision of extracorporeal $\mathrm{CO}_{2}$ removal concomitantly with CRRT has been investigated in the management of patients with multisystem organ failure by positioning a commercial hollow-fiber membrane oxygenator in parallel with the hemofilter of a CRRT system. ${ }^{33}$ Quintard et $\mathrm{al}^{37}$ applied this strategy in 16 subjects who were receiving CRRT for kidney dysfunction and developed hypercapnic respiratory acidosis. This was accomplished with the addition of a Medos Hilite LT membrane (Xenios) prior to the hemofilter of a Multifiltrate system (Fresenius, Bad Homburg, Germany). In this series, $\mathrm{P}_{\mathrm{aCO}}$ was reduced by $31-39 \%$ over a 12 -h period, with a $\mathrm{pH}$ improvement of 0.23 .

This approach was also piloted in a series of 10 subjects with combined respiratory and renal dysfunction utilizing the Polyflux $140 \mathrm{H}$ system (Gambro, Hechingen, Germany) plus a D902 Lilliput 2 membrane (LivaNova, Italy) placed after the dialysis filter, which was described as lung-assisting renal replacement system. ${ }^{38}$ Blood flows were $250-500 \mathrm{~mL} / \mathrm{min}$ with sweep gas flows of $4-6$ $\mathrm{L} / \mathrm{min}$, resulting in a $28 \%$ decrease in $\mathrm{P}_{\mathrm{aCO}}$ and a corresponding $\mathrm{pH}$ increase of 0.12 . In addition, subjects 
had improved hemodynamics as demonstrated by the ability to wean them from vasoactive agents, and there were no technical issues related to the functioning of the CRRT system.

\section{Wearable ECMO}

ECMO systems with compact designs and integral components, along with the development of a bi-caval duallumen cannula, have extended the use of ECMO to patients awaiting lung transplantation. ${ }^{39}$ This paradigm shift to longer-term support has prompted a different approach to the clinical management of these patients including liberation from mechanical ventilation, ambulation, and other rehabilitative care. ${ }^{40}$ There are a number of successful accounts of this approach, which have stimulated interest in developing miniaturized systems that could better facilitate ambulation and movement, and thus potentially extend ECMO duration for other chronic respiratory conditions. ${ }^{41}$

The portable pediatric pump-lung is a compact, integrated, microporous, hollow-fiber membrane oxygenator and centrifugal pump that weighs approximately $280 \mathrm{~g}$, has a blood surface area of $0.3 \mathrm{~m}^{2}$, and has a priming volume slightly over $100 \mathrm{~mL} .{ }^{42}$ The fibers in the membrane are arranged in a unique circumferential-radial pattern, and the pump is a magnetically levitated rotating impeller driven by a motor. This device was used in a juvenile sheep model to determine whether support could be sustained for $30 \mathrm{~d}$ and to assess biocompatibility and gas transfer. ${ }^{42}$ The pediatric pump-lung was implanted in 8 sheep with the apparatus in a harness on the animal's back. The pump operated at around 2,500 revolutions/min to achieve blood flows of $1-1.5 \mathrm{~L} / \mathrm{min}$, and oxygen transfer rates were approximately $50-65 \mathrm{~mL} / \mathrm{min}$. Results from this preliminary study are encouraging as support was maintained for $30 \mathrm{~d}$ and there was minimal impact on hematologic and blood chemistry parameters. Developers of this device hope to provide an alternative for long-term mechanical circulatory support for infants and children with severe cardiorespiratory failure, as candidacy for organ transplantation is determined.

Additional experiments with the pediatric pump-lung were conducted in a sheep model of acute respiratory failure to further evaluate the gas-exchange capabilities. ${ }^{43} \mathrm{Re}$ spiratory failure was induced in 6 animals after placement of the pediatric pump-lung. As soon as hypoxemic and hypercapnic indices of respiratory failure were attained and changes in hemodynamic parameters occurred, blood and sweep gas flow to the pediatric pump-lung was initiated. When support with the pediatric pump-lung was established, blood gas derangements corrected, hemodynamics stabilized, and right ventricular work decreased.

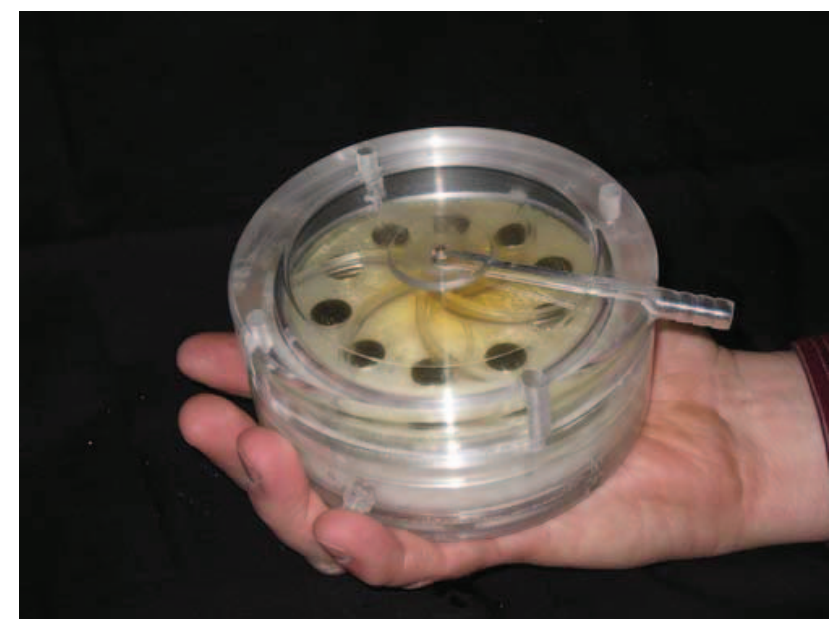

Fig. 9. The paracorporeal ambulatory assist lung prototype. Courtesy of the University of Pittsburgh.

Integrating hollow-fiber membranes with centrifugal pumps greatly reduces the size and priming volume of an ECMO system to a truly compact and wearable size. The paracorporeal ambulatory assist lung, a small device weighing $1.8 \mathrm{~kg}$ and with dimensions of roughly $13 \times 13 \times 13 \mathrm{~cm}$, is being developed for this purpose (Fig. 9). ${ }^{44}$ The stacktype fiber bundles and the impellers with embedded magnets allow the miniaturized profile. In vitro and in vivo testing has demonstrated that the paracorporeal ambulatory assist lung generated flows of $3.5 \mathrm{~L} / \mathrm{min}$ at rotational speed of 2,100 revolutions/min and provided sufficient $\mathrm{O}_{2}$ and $\mathrm{CO}_{2}$ transfer.

Two pumpless devices intended for long-term use are being developed at the University of Michigan: the Mlung and a compliant thoracic artificial lung. ${ }^{45}$ The M-lung contains blood and gas paths that pass through a series of fiber-bundle compartments arranged so that blood and gas flow run perpendicular to each other. Computational flow dynamics and in vivo testing of this low-resistance device demonstrated that the mixing of blood as it traversed the gated compartments augmented $\mathrm{O}_{2}$ transfer and sufficiently removed $\mathrm{CO}_{2}$ at a rated flow of $2 \mathrm{~L} / \mathrm{min} .{ }^{46}$ The compliant thoracic artificial lung consists of a flexible chamber containing a mat of hollow-fiber bundles through which blood is routed by means of inlet and outlet conduits, while fresh gas flows adjacently. ${ }^{47}$ Initial animal experiments with this device showed that the load on the right ventricle could be reduced with the device positioned between the pulmonary artery and left atrium, and flows $>7 \mathrm{~L} / \mathrm{min}$ could be achieved. Additional studies demonstrated that the device could be maintained for $14 \mathrm{~d}$ without any appreciable increase in resistance, while maintaining normal physiologic function and with minimal thrombus formation. ${ }^{45}$ With further refinements and evaluated over longer durations, these thoracic artificial lung prototypes may be a viable 
alternative for the support of patients awaiting lung transplantation.

\section{Simulation-Based Training}

The use of simulation-based training has become a valuable tool in the preparation of medical and surgical teams, enhancing team functionality, safety, surgical technique, and knowledge. ${ }^{48-50}$ ECMO programs have incorporated simulation-based training into their curricula and as a mechanism for skills enhancement, team development, improving clinical outcomes, and maintaining competency. ${ }^{51-53}$ The success of simulation-based training is influenced by the technology used to create clinical and physiologic realism for routine and emergency ECMO scenarios.

Modifications to simulation mannequins have been one approach, such as the reusable model proposed by Thompson in which tubes positioned in the neck serve as surrogates for the internal jugular vein and carotid vessels, which are then overlaid with a simulated skin. ${ }^{54}$ This model was found to sufficiently replicate cannulation steps and bring greater fidelity to the simulation experience, which was perceived to improve team work and participant confidence. Another mannequin modification was reported by Allan et al, ${ }^{55}$ consisting of an imbedded skills trainer developed in conjunction with cardiovascular surgeons. The haptic trainer was designed to mimic skin and tissue layers such that vessel identification was representative of a real incision and dissection involved anatomic accuracy and bleeding (Fig. 10). Cardiothoracic surgeons participating in an ECMO cannulation skills program using this trainer were found to have improved skills and overall greater proficiency. Similarly, an open-chest model was developed and used to simulate scenarios such as cardiac tamponade and the effect on ECMO flow. ${ }^{56}$ This system was found to be beneficial to end-users, with enhancement in both skills and communication.

The modification of simulation mannequins is one step toward greater fidelity in ECMO simulation-based training. Further authenticity could be achieved with the ability to concomitantly alter mannequin physiology and ECMO circuit and system response. Ribeiro et $\mathrm{al}^{57}$ created a realistic model utilizing a harvested porcine heart, a ventricular assist device, and the Califia cardiopulmonary bypass simulator developed by Biomed Simulation (Poway, California). The porcine heart was prepared with surrogate inferior vena cava and aorta, which were mechanized with a ventricular assist device to mimic a beating heart. Additional lines were directed to the Califia simulator, which controlled pressurization of the heart. The heart was placed in the thorax of a mannequin to create an innovative and authentic platform for the cannulation and application of cardiopulmonary bypass.

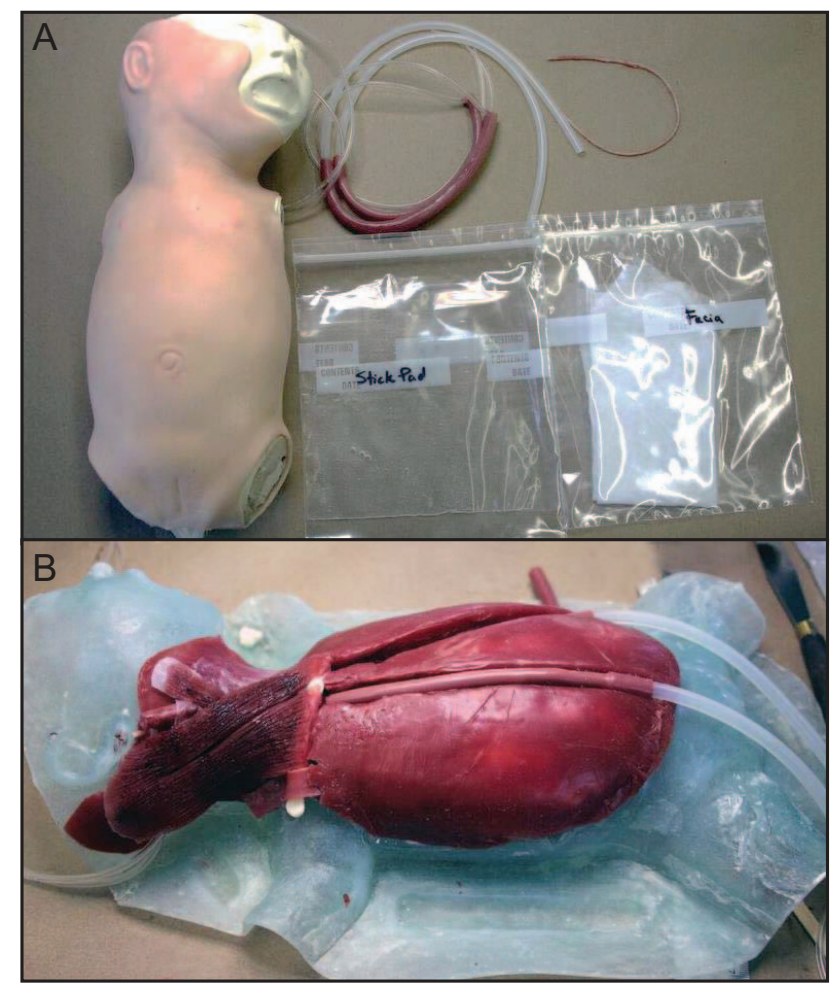

Fig. 10. Imbedded haptic skills trainer kit (A) used for surgical placement of extracorporeal membrane oxygenation cannulae during simulation training (B). Courtesy of Dr Peter Weinstock, Boston Children's Hospital Simulator Program.

The EigenFlow (Curtis Life Research, Indianapolis, Indiana) is a stand alone simulator used to create various conditions during ECMO, such as decreased venous drainage, air emboli, or changes to transmembrane pressure. The device is spliced into an ECMO circuit and is remotely controlled through wireless technology, which permits the user to readily alter flow and pressures to create a more dynamic experience with varying degrees of acuity simulated.

The use of mannequin-based simulation training has become prevalent in ECMO centers and is an important adjunct to training methods recommended by the Extracorporeal Life Support Organization. ${ }^{5}$ The development of ECMO-ready mannequins that can be surgically accessed and programmed to create physiologic and clinical realism remains an unmet need. Devices like the Califia, while aimed at cardiopulmonary bypass simulation exercises, may have a role in creating physiologic variations associated with ECMO and might create a more authentic experience for learners. The coalescence of a mannequin and programmable ECMO system that can be remotely altered may achieve greater realism during troubleshooting sequences and clinical management of an ECMO patient. 


\section{Technical Advances in ECMO}

\section{Summary}

Technical improvements to ECMO components such as plasma-tight PMP diffusion membranes and pump-membrane combinations, along with the development of integrated systems, have allowed for expanded indications and improved safety. It is intriguing to think that less invasive technology can be developed, perhaps into a single compact platform, to which cartridge-type components may be added to manage specific organ dysfunction, such as the combined renal-pulmonary dialysis concept. Ongoing research is needed to determine whether wearable thoracic artificial lungs can move from bench to bedside, along with parallel studies to determine optimal biocompatibility. Identifying target populations through rigorous studies is also needed to determine which technological advancements will enhance wellness and improve outcomes. Enhanced and more realistic simulation-based training can be established with ongoing innovation so that the specialists who manage new devices and the clinical teams who manage the patients will gain even greater proficiency.

\section{REFERENCES}

1. Thiagarajan RR, Barbaro RP, Rycus PT, McMullan M, Conrad SA, Fortenberry JD, Paden ML. Extracorporeal life support organization registry international report 2016. ASAIO J 2017;63(1):60-67.

2. Abrams D, Brodie D. Extracorporeal membrane oxygenation for adult respiratory failure: 2017 update. Chest 2017;152(3):639-649.

3. Müller T, Bein T, Phillip A, Graf B, Schmid C, Riegger G. Extracorporeal pulmonary support in severe pulmonary failure in adults. Dtsch Arztebl Int 2013;110(10):159-166.

4. Toomasian JM, Schreiner RJ, Meyer DE, Schmidt ME, Hagan SE, Griffith GW, et al. A polymethylpentene fiber gas exchanger for long-term extracorporeal life support. ASAIO J 2005;51(4):390-397.

5. Weems MF, Friedlich PS, Nelson LP, Rake AJ, Klee L, Stein JE, Stavroudis TA. The role of extracorporeal membrane oxygenation simulation training at extracorporeal life support organization centers in the United States. Sim Healthcare 2017;12(3):233-239.

6. Yeager T, Shuvo R. Evolution of gas permeable membranes for extracorporeal membrane oxygenation. Artificial Organs 2017;41(8): 700-709.

7. Strueber M. Artificial lungs: are we there yet? Thorac Surg Clin 2015;25(1):107-113.

8. Eash HJ, Jones HM, Hattler BG, Federspiel WJ. Evaluation of plasma resistant hollow fiber membranes for artificial lungs. ASAIO J 2004; 50(5):491-497.

9. Rambaud J, Guilbert J, Guellec I, Renolleau S. A pilot study comparing two polymethylpentene extracorporeal membrane oxygenators. Perfusion 2012;218(1):14-20.

10. Gill MC, O'Shaughnessy K, Dittmer J. A paedatric ECMO case of plasma leakage through a polymethylpentene oxygenator. Perfusion 2015;30(7):600-603.

11. O'Brien C, Monteagudo J, Schad C, Cheung E, Middlesworth W. Centrifugal pumps and hemolysis in pediatric extracorporeal membrane oxygenation (ECMO) patients: an analysis of Extracorporeal Life Support (ELSO) registry data. J Pediatr Surg 2017;52(6):975978.

12. Lawson DS, Lawson AF, Walczak R, McRobb C, McDermott P, Shearer IR, et al. North American neonatal extracorporeal membrane oxygenation (ECMO) devices and team roles: 2008 survey results of Extracorporeal Life Support Organization (ELSO) centers. Extra Corpor Technol 2008;40(3):166-174.

13. Alwardt CM, Wilson DS, Alore ML, Lanza LA, Devaleria PA, Pajaro OE. Performance and safety of an integrated portable extracorporeal life support system for adults. J Extra Corpor Technol 2015; 47(1):38-43.

14. Haneya A, Philipp A, Foltan M, Camboni D, Müeller T, Bein T, et al. First experience with the new portable extracorporeal membrane oxygenation system Cardiohelp for severe respiratory failure in adults. Perfusion 2012;27(2):150-155.

15. Philipp A, Arlt M, Amann M, Lunz D, Müller T, Hilker M, et al First experience with the ultra-compact mobile extracorporeal membrane oxygenation system Cardiohelp in interhospital transport. Interact Cardiovasc Thorac Surg 2011;12(6):978-981.

16. Fleck T, Benk C, Klemm R, Kroll J, Siepe M, Grohmann J, et al First serial in vivo results of mechanical circulatory support in children with a new diagonal pump. Eur J Cardiothorac Surg 2013;44(5): 828-835.

17. Lunz D, Phillip A, Judeman K, Amann M, Foltan M, Schmid C, et al. First experience with the Deltastream DP3 in venovenous extracorporeal membrane oxygenation and air-supported inter-hospital transport. Interact CardioVasc Thorac Surg 2013;17(5):773777.

18. Stiller B, Houmes RJ, Rüffer A, Kumpf M, Müller A, Kipfmüller F, et al. Multicenter experience with mechanical circulatory support using a new diagonal pump in 233 children. Artif Organs 2018; 42(4):377-385.

19. Speth M, Münch F, Purbojo A, Glöckler M, Toka O, Cesnjevar RA, Rüffer A. Pediatric extracorporeal life support using a third generation diagonal pump. ASAIO J 2016;62(4):482-490.

20. Evenson A, Wang S, Kunselman AR, Ündar A. Use of a novel diagonal pump in an in vitro neonatal pulsatile extracorporeal life support circuit. Artificial Organs 2014;38(1):E1-E9.

21. Wang S, Kunselman AR, Clark JB, Ündar A. In vitro hemodynamic evaluation of a novel pulsatile extracorporeal life support system: impact of perfusion modes and circuit components on energy loss. Artif Organs 2015;39(1):59-66.

22. Wang S, Kunselman AR, Ündar A. Novel diagonal pump for pediatric extracorporeal life support system. Artificial Organs 2013;37(1): $37-47$

23. Patel S, Wang S, Pauliks L, Chang D, Clark JB, Kunselman AR, Ündar A. Evaluation of a novel pulsatile extracorporeal life support system synchronized to the cardiac cycle: effect of rhythm changes on hemodynamic performance. Artif Organs 2015;39(1):67-76.

24. Cove ME, MacLaren G, Federspiel WJ, Kellum JA. Bench to bedside review: extracorporeal carbon dioxide removal, past, present and future. Critical Care 2012;16(5):232-241.

25. Hermann A, Staudinger T, Bojic A, Riss K, Wohlfarth P, Robak O, et al. First experience with a new miniaturized pump-driven venovenous extracorporeal $\mathrm{CO}_{2}$ removal system (iLA Activve): a retrospective data analysis. ASAIO J. 2014;60(3):342-347.

26. Hermann A, Riss K, Schellongowski P, Bojic A, Wohlfarth P, Robak $\mathrm{O}$, et al. A novel pump-driven veno-venous gas exchange system during extracorporeal CO2-removal. Intensive Care Med 2015; 41(10):1773-1780

27. Braune S, Sieweke A, Brettner F, Staudinger T, Joannidis M, Verbrugge $\mathrm{S}$, et al. The feasibility and safety of extracorporeal carbon dioxide removal to avoid intubation in patients with COPD unresponsive to noninvasive ventilation for acute hypercapnic respiratory failure (ECLAIR study): multicentre case-control study. Intensive Care Med 2016;42(9):1437-1444.

28. Jeffries RG, Mussin Y, Bulanin DS, Lund LW, Kocyildirim E, Zhumadilov ZZ, et al. Pre-clinical evaluation of an adult extracorporeal 


\section{Technical Advances in ECMO}

carbon dioxide removal system with active mixing for pediatric respiratory support. Int J Artif Organs 2014;37(12):888-899.

29. Bermudez CA, Zaldonis D, Fan MH, Pilewski JM, Crespo MM. Prolonged use of the hemolung respiratory assist system as a bridge to redo lung transplantation. Ann Thorac Surg 2015;100(6):23302333.

30. Burki NK, Mani RK, Herth FJF, Schmidt W, Teschler H, Bonin F, et al. A novel extracorporeal $\mathrm{CO}_{2}$ removal system: results of a pilot study of hypercapnic respiratory failure in patients with COPD. Chest 2013;143(3):678-686.

31. Tiruvoipati R, Buscher H, Winearls J, Breeding J, Ghosh D, Chaterjee $\mathrm{S}$, et al. Early experience of a new extracorporeal carbon dioxide removal device for acute hypercapnic respiratory failure. Crit Care Resusc 2016;18(4):261-269.

32. Fanelli V, Ranieri MV, Mancebo J, Moerer O, Quintel M, Morley S, et al. Feasibility and safety of low-flow extracorporeal carbon dioxide removal to facilitate ultra-protective ventilation in patients with moderate acute respiratory distress syndrome. Crit Care 2016;20:36-43.

33. Romagnoli S, Ricci Z. Ronco C. Novel extracorporeal therapies for combined renal-pulmonary dysfunction. Semin Nephrol 2016;36(1): 71-77.

34. Godet T, Combes A, Zogheib E, Jabaudon M, Futier E, Slutsky AS, Constantin JM. Novel $\mathrm{CO}_{2}$ removal device driven by a renal-replacement system without hemofilter: a first step experimental validation. Anaesth Crit Care Pain Med 2015;34(3):135-140.

35. Eloot S, Peperstraete H, DeSomer F, Hoste E. Assessment of the optimal operating parameters during extracorporeal $\mathrm{CO}_{2}$ removal with the Abylcap® system. Int J Artif Organs 2016;39(11):580-585.

36. Peperstraete H, Eloot S, Depuydt P, DeSomer F, Hoste E, Roosen C, Hoste E. Low flow extracorporeal $\mathrm{CO}_{2}$ removal in ARDS patients: a prospective short-term crossover pilot study. BMJ Anesthesiology 2017;17(1):155-164.

37. Quintard JM, Barbot O, Thevenot F, de Matteis O, Benayoun L, Leibinger F. Partial extracorporeal carbon dioxide removal using a standard continuous renal replacement therapy device: a preliminary study. ASAIO J 2014;60(5):564-569.

38. Forster C, Schriewer J, John S, Eckardt KU, Willam C. Low-flow $\mathrm{CO}_{2}$ removal integrated into a renal-replacement circuit can reduce acidosis and decrease vasopressor requirements. Crit Care 2013; 17(4):R154.

39. Rehder KJ, Turner DA, Hartwig MG, Williford WL, Bonadonna D, Walczak RJ Jr., et al. Active rehabilitation during extracorporeal membrane oxygenation as a bridge to lung transplantation. Respir Care 2013;58(8):1291-1298.

40. Bain JC, Turner DA, Rehder KJ, Eisenstein EL, Davis RD, Cheifetz IM, Zaas DW. Economic outcomes of extracorporeal membrane oxygenation with and without ambulation as a bridge to lung transplantation. Respir Care 2016;61(1):1-7.

41. Spinelli E, Protti A. Get fit for lung transplant with ambulatory extracorporeal membrane oxygenation! Respir Care 2016;61(1):117-118.

42. Liu Y, Sanchez PG, Wei X, Watkins AC, Niu S, Wu ZJ, Griffith BP. Effects of cardiopulmonary support with a novel pediatric pump-lung in a 30-day ovine animal model. Artif Organs 2015;39(12):989-997.

43. Wei X, Sanchez PG, Liu Y, Watkins AC, Li T, Griffith BP, Wu ZJ. Extracorporeal respiratory support with a miniature integrated pedi- atric pump-lung device in an acute ovine respiratory failure model. Artif Organs 2016;40(11):1046-1053.

44. Madhani SP, Frankowski BJ, Burgreen GW, Antaki JF, Kormos R, D'Cunha J, Federspiel WJ. In vitro and in vivo evaluation of a novel integrated wearable artificial lung. J Heart Lung Transplant 2017; 36(7):806-811.

45. Skoog DJ, Pohlmann JR, Demos DS, Scipione CN, Iyengar A, Schewe RE, et al. Fourteen day in vivo testing of a compliant thoracic artificial lung. ASAIO J 2017;63(5):644-649.

46. Fernando UP, Thompson AJ, Potkay J, Cheriyan H, Toomasian J, Kaesler A, et al. A membrane lung design based on circular blood flow paths. ASAIO J 2017;63(5):637-643.

47. Schewe RE, Khanafer KM, Arab A, Mitchell JA, Skoog DJ, Cook KE. Design and in vitro assessment of an improved, low-resistance, compliant thoracic artificial lung. ASAIO J 2012 58(6):583-589.

48. Beal MD, Kinnear J, Anderson CR, Martin TD, Wamboldt R, Hooper $\mathrm{L}$. The effectiveness of medical simulation in teaching medical students critical care medicine: a systematic review and meta-analysis. Simul Healthcare 2017;12(2):104-116.

49. Allan CK, Thiagarajan RR, Beke D, Imprescia A, Kappus LJ, Garden A, et al. Simulation-based training delivered directly to the pediatric cardiac intensive care unit engenders preparedness, comfort, and decreased anxiety among multidisciplinary resuscitation teams. J Thorac Cardiovasc Surg 2010;14D(3)140:645-652.

50. Smelt JLC, Phillips S, Hamilton C, Fricker P, Spray D, Nowell JL, Jahangiri M. Simulator teaching of cardiopulmonary bypass complications: a prospective, randomized study. J Surg Ed 2016;73(6):10261031.

51. Fehr JJ, Shepard M, McBride ME, Mehegan M, Reddy K, Murray DJ, Boulet JR. Simulation-based assessment of ECMO clinical specialists. Sim Healthcare 2016;11(3):194-199.

52. Sanchez-Glanville C, Brindle ME, Spence T, Blackwood J, Drews T, Menzies S, Lopushinsky SR. Evaluating the introduction of extracorporeal life support technology to a tertiary-care pediatric institution: smoothing the learning curve through interprofessional simulation training. J Pediatr Surg 2015;50(5):798-804.

53. Zakhary BM, Kam LM, Kaufman BS, Felner KJ. The utility of high-fidelity simulation for training critical care fellows in the management of extracorporeal membrane oxygenation emergencies: a randomized controlled trial. Crit Care Med 2017;45(8):1367-1373.

54. Thompson JL, Grisham LM, Scott J, Mogan C, Prescher H, Biffar $\mathrm{D}$, et al. Construction of a reusable, high-fidelity model to enhance extracorporeal membrane oxygenation training through simulation. Adv Neonatal Care 2014;14(2):103-109.

55. Allan CK, Pigula F, Bacha EA, Emani S, Fynn-Thompson F, Thiagarajan RR, et al. An extracorporeal membrane oxygenation cannulation curriculum featuring a novel integrated skills trainer leads to improved performance among pediatric cardiac surgery trainees. Sim Healthcare 2013;8(4):221-228.

56. Atamanyuka I, Gheza O, Saeeda I, Laneb M, Hall J, Jackson T, et al. Impact of an open-chest extracorporeal membrane oxygenation model for in situ simulated team training: a pilot study. Interact Cardiovasc Thorac Surg 2014;18(1):17-20.

57. Ribeiro IB, Ngu JMC, Gurinder G, Rubens FD. Development of a high fidelity beating heart simulator for cardiac surgery training. Perfusion 2017;32(7):568-573. 